\title{
Echocardiographic assessment of the left atrial function in patients with mitral valve disease by two-dimensional speckle tracking
}

Hussein Awad Hemeda*, Mahmoud Shawky Abd Elmoneum, Eman Said Al Kishk and Sayed Abd El Khaleq El Darky

*Correspondence: husseine86@gmail.com

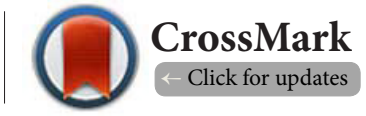

Cardiology Department, Benha University Hospital, Benha city, Egypt.

\begin{abstract}
Objectives: Comparing effects of mitral stenosis and mitral regurgitation on LA deformation using twodimensional speckle tracking echocardiography.

Methods: Fifty patients with isolated moderate to severe MS, fifty patients with isolated moderate to severe MR and normal left ventricular ejection fraction (EF $\geq 55 \%)$ were compared using one way analysis of variance (ANOVA) test with two-group comparisons. LA reservoir strain was calculated as peak systolic atrial longitudinal strain, LA reservoir strain rate as peak systolic positive value, LA conduit SR as the early diastolic negative peak, and LA contractile SR as the late diastolic negative peak with QRS onset as the reference point.

Results: There was a statistically significant difference between the LA average PALS ( $<<0.001)$, SRS $(\mathrm{p}=0.012)$, SRE $(\mathrm{p}<0.001)$, TTP strain $(\mathrm{p}<0.001)$, SRA $(\mathrm{p}=0.010)$, and TTP SRE $(\mathrm{p}=0.005)$ of the 2 patient groups. LA average PALS was significantly higher in MR compared to MS patients $(p<0.001)$.The average SRS, SRE and SRA were nearly similar in MS and MR patients. The TTP strain and TTP SRE were significantly shorter in MR compared to MS patients $(p<0.001$ and $p=0.005$, respectively). Significant inverse correlations were noted between the LA volume index (LAVI) and average PALS ( $\mathrm{r}=-0.898$, $\mathrm{P}<0.001)$, SRS ( $\mathrm{r}=-0.927, \mathrm{P}<0.001), \mathrm{SRE}(\mathrm{r}=-0.938, \mathrm{P}<0.001)$ and $\mathrm{SRA}(\mathrm{r}=-0.957, \mathrm{P}<0.001)$ in MR patients.

Conclusion: Left atrial PALS, TTP strain, and TTP SRE can differentiate between volume overloaded and pressure overloaded left atrium. Pressure overload seems to have a more pronounced effect on LA mechanics.
\end{abstract}

Keywords: Speckle tracking, mitral stenosis, mitral regurgitation, strain, strain rate, left atrium

\section{Introduction}

Rheumatic heart disease (RHD) remains the leading acquired heart disease in the young, causing $>350,000$ premature cardiovascular deaths each year worldwide [1]. The advent of echocardiographic screening in endemic areas has led to a new epidemiological pyramid-type model combining subclinical RHD at the base and symptomatic RHD leading to hospital admission at the top [2].

The most common cause of MS worldwide is post-rheumatic valvular deformity. This is evident in the high prevalence of rheumatic heart disease in developing nations. However, in developed nations, DMS represents a sizeable proportion of patients with MS. The other less common causes of MS include congenital deformities, mucopolysaccharidosis, systemic disorders such as systemic lupus erythematosus, Whipple disease, and disorders associated with abnormal serotonin metabolism [3].

Organic MR refers to primary leaflet abnormality resulting in MR. The cause is most often rheumatic in South African (SA) patients, whereas degenerative or myxomatous disease is most common in the developed world. Other causes include fibroelastic disease, congenital (isolated cleft or atrioventricular (AV) canal defect), Marfan syndrome and endocarditis [4].

Traditionally, assessment of left atrial function has been performed by measuring volumes with $2 \mathrm{D}$ echocardiography. 
Hemeda et al. Cardiovascular System 2018,

Additionally, it can be assessed with transmitral Doppler and pulmonary vein Doppler. Recently, an alternative method has been incorporated, namely, measurement of myocardial deformation with color tissue Doppler-derived strain [5].

STE is a new technique of 2D-echocardiography image analysis that allows the study of regional atrial myocardial deformation expressed by a dimensionless parameter, the strain, which is defined as the percentage change from the original dimension [6].

\section{Patients and methods}

This study had been conducted in Cardiology Department Faculty of Medicine, Benha University) Egypt, from May 2017 to March 2018. The study population has been randomly selected from the cardiology department and outpatient clinic. The patients diagnosed with mitral valve disease were included in the study. Patients were divided into 2 arms equally, the first arm included fifty patients with mitral stenosis of rheumatic origin, the second arm included fifty patients with mitral regurgitation of rheumatic origin or myxomatous origin (mitral valve prolapsed). Exclusion criterion was patient refusal, with prosthetic mitral valve, double MVD, any rhythm other than sinus rhythm, congenital heart disease, aortic valve disease and chronic secondary MR. An informed consent was obtained from the patients or their legal guardians after approval of the Ethical Committee of Benha University of Medical Science. The work complies with the principles of the Declaration of Helsinki in 1964.

All study population underwent the following diagnostic work up:

1. Full medical history including (Age, sex, symptoms suggestive of cardiac disease and current medications .

2. Twelve lead electrocardiogram (ECG) to exclude established AF and to measure PR interval and p-wave morphology to exclude heart block of any degree or nodal rhythm.

3. Standard resting transthoracic echocardiography (TTE): A MyLab60 echo machine 2009 equipped with a multifrequency 2.5-3.5 MHz transducer was used to evaluate the following: LA dimensions, LV dimensions, LV ejection fraction and severity of MS or MR.

4. Speckle tracking Echocardiography (STE): Apical four chamber view images were obtained using conventional two-dimensional gray scale echocardiography during breath holding and with a stable ECG recording. Three consecutive heart cycles were recorded and analyzed. Recordings were processed off line using commercially available semi-automated two-dimensional strain software (Mylab 60, Esaote, S. p, A-Italy). Left atrial endocardial surface was manually traced in four chamber views in end systole with the possibility of further correction. The software generated the following measurements automatically:

A. Left atrial volume: Left atrial Volume index was calculated by the formula:

\section{LAVmax/BSA}

Where LAVmax is Maximum left atrial volume and BSA is the body surface area $[7,8]$.

B. Left atrial emptying fraction (LAEF).

C. Strain curve for each atrial segment in apical four chamber view from which we obtained Peak atrial longitudinal strain (PALS) during systole and Time to peak atrial longitudinal strain (TTP Strain).

Regarding the measurement of peak atrial strain, as stated in the current ASE/EAE Consensus, two techniques have been proposed to quantify atrial deformation by STE, which differ only by the choice of frame from which to start processing software. The first takes as reference point the QRS onset and measures the positive peak atrial longitudinal strain, corresponding to atrial reservoir, the second uses the $\mathrm{P}$ wave as the reference point, enabling the measurement of a first negative peak atrial longitudinal strain, corresponding to atrial systole, a second positive peak atrial strain, corresponding to LA conduit function, and their sum [9].

D.Strain rate curve for each atrial segment in apical four chamber view from which we obtained: Strain rate S (SR S), Strain rate E (SR E), Strain rate A (SR A),Time to peak strain rate $S$ (TTP SR S), Time to peak strain rate E (TTP SR E), Time to peak strain rate $A$ (TTP SR A).

To measure Strain and strain rate and time to peak strain and strain rate of left atrial walls, offline computer-based analysis for strain and SR were performed using XStrain software based on a feature tracking algorithm. The LA wall was divided automatically into six segments from septal mitral annulus to lateral mitral annulus in the apical four chamber view, segment one and two corresponding to the interatrial septum, segment three and four to the roof of the left atrium and five and six to the lateral wall. Because there is still no software available to calculate atrial strain, we employed the same software used for the analysis of ventricular function. As a result, the peak strain automatically chosen by the software (Mylab 60, Esaote, S. p, A-Italy) was at the peak of the QRS so it had to be manually readjusted to the PALS and that was also the case with SR S, SR E and SR A which were all manually measured.

All the average values of strain, strain rate, and time to peak were calculated by averaging values observed in all six LA segments in four chamber view.

E. The PA-interval: We measured it from the onset of P-wave of the ECG to the peak of A wave, the A wave was that of longitudinal velocity of the basal segment of the left atrial lateral wall generated by speckle tracking technique. It was measured from $P$ to peak $A$ for the sake of accuracy as the onset of the wave was not obvious all the time.

PA interval was measured by Quintana et al., as the time difference from onset of $P$ wave to the beginning, peak and end of A wave at several right and left atrial walls, to assess time to atrial activation [10], and the A wave was that of tissue Doppler imaging. 


\section{Statistical analysis}

Data were statistically described in terms of mean \pm standard deviation $( \pm S D)$, and range, or frequencies (number of cases) and percentages when appropriate. Comparison of numerical variables between the study groups was done using one way analysis of variance (ANOVA) test with post-hoc multiple 2-group comparisons. For comparing categorical data, Chi square (X2) test was performed. Exact test was used instead when the expected frequency is less than 5 . Correlation between various variables was done using Pearson moment correlation equation for linear relation in normally distributed variables and Spearman rank correlation equation for non-normal variables. $P$ values less than 0.05 was considered statistically significant. All statistical calculations were done using computer program SPSS (Statistical Package for the Social Science; SPSS Inc., Chicago, IL, USA) release 15 for Microsoft Windows (2006).

\section{Results}

\section{Demographic characteristics}

Fifty mitral Stenosis (MS) patients and fifty mitral regurgitation (MR) patients were included in the study. As shown in (Table 1), There was no significant statistical difference (NS) in Gender, Age or BSA between MS and MR groups ( $p>0.05$ ).

\section{Echocardiographic data}

\section{Severity of valvular lesion}

As shown in (Table 2), the MR group had 4 patients with moderate lesion and 46 patients with severe lesion, the MS group had 7 patients with moderate lesion $(M V A \leq 1.5 \mathrm{~cm} 2)$ and 43 patients with severe lesion (MVA $\leq 1.0 \mathrm{~cm} 2)$ with no statistical significance ( $p$-value $=0.338$ ).

\section{Heart rate and Left ventricular function}

All patients were in sinus rhythm. As shown in (Table 3), the two study groups were similar regarding the heart rate. They were also similar in terms of global LV function as measured by LVEF with no statistical significant difference. None of the patients included had systolic function impairment.

Table 1. Demographic characteristics of two groups.

\begin{tabular}{llll}
\hline & MS $(\mathbf{n}=\mathbf{5 0})$ & MR $(\mathbf{n}=\mathbf{5 0})$ & p value \\
\hline Age, years & & & \\
\hline Mean \pm SD (range) & $33.100 \pm 7.823$ & $35.180 \pm 8.705$ & 0.212 \\
\hline BSA (m2) & & & \\
\hline Mean \pm SD (range) & $1.706 \pm 0.135$ & $1.662 \pm 0.119$ & 0.087 \\
\hline Sex & & & \\
\hline Male & $13(26 \%)$ & $13(26 \%)$ & 1.000 \\
Female & $37(74 \%)$ & $37(74 \%)$ & \\
\hline
\end{tabular}

Data are presented as mean \pm SD or number (percent), $B S A=$ body surface area, $\mathrm{MR}=$ mitral regurgitation, $\mathrm{MS}=$ mitral stenosis, $\mathrm{N}=$ number
Table 2. Distribution of the mitral valve disease patients according to severity of the lesion.

\begin{tabular}{|c|c|c|c|c|c|c|c|c|}
\hline \multirow{3}{*}{$\begin{array}{l}\text { Mitral } \\
\text { valve } \\
\text { disease }\end{array}$} & \multicolumn{6}{|c|}{ Groups } & \multicolumn{2}{|c|}{ Chi-Square } \\
\hline & \multicolumn{2}{|c|}{ MR } & \multicolumn{2}{|c|}{ MS } & \multicolumn{2}{|c|}{ Total } & \multirow[b]{2}{*}{$\mathrm{X}^{2}$} & \multirow[b]{2}{*}{ P-value } \\
\hline & $\mathbf{N}$ & $\%$ & $\mathbf{N}$ & $\%$ & $\mathbf{N}$ & $\%$ & & \\
\hline Moderate & 4 & 8.00 & 7 & 14.00 & 11 & 11.00 & 0.919 & 0.338 \\
\hline Severe & 46 & 92.00 & 43 & 86.00 & 89 & 89.00 & & \\
\hline Total & 50 & 100.00 & 50 & 100.00 & 100 & 100.00 & & \\
\hline
\end{tabular}

Data are presented as mean \pm SD or number (percent).

Table 3. Heart rate (HR) and left ventricular ejection fraction (LVEF) in the two groups.

\begin{tabular}{lllll}
\hline & \multicolumn{2}{c}{ Groups } & \multicolumn{3}{c}{ T-Test } \\
\cline { 2 - 3 } & MR & & \\
\cline { 2 - 3 } & Mean \pm SD & Mean \pm SD & T & P-value \\
\hline HR $($ bpm) $)$ & $81.070 \pm 13.442$ & $77.014 \pm 13.193$ & 1.523 & 0.131 \\
LVEF \% & $62.504 \pm 9.551$ & $65.122 \pm 4.604$ & -1.746 & 0.084 \\
\hline
\end{tabular}

Data are presented as mean \pm SD or number (percent).

3-Left atrial size, Left atrial volume index(LAVI), left atrial area change and left atrial ejection fraction (LAEF): As shown in (Table 4); Regarding left atrial diameter There was no statistically significant difference between the two groups in the atrial diameter ( $p$-value $=0.608$ ), Left atrial volume index (LAVI) was significantly higher in the MR group than MS group ( $p$-value $<0.05$ ), there was a statistically significant difference between the two groups in the atrial area change, Patients with MR having the higher value and those with MS having the lower value ( $p$ value $<0.001$ ) and lastly there was a statistically significant difference between the two groups in Atrial EF as patients with MR having the higher value and the MS group having the lower value ( $p$ value $<0.001$ ).

\section{Speckle Tracking echocardiography}

1-P-A interval: There was a statistically significant difference among the two groups with the lower value being that of MR group and the higher being that of the MS group, with ( $p$ value $=0.002$ ) (Table 5$)$.

2- Strain and Strain rate parameters (Table 6); In Average PALS, There was statistically significant difference in the two groups, where the value was lower in MS group and higher in MR group with ( $p$ value $<0.001$ ). In Average SR S, Average SR E and Average SR A, There was statistically significant difference in the two groups, where their values were lower in MS group and higher in MR group with ( $p$ value $<0.05$ ), There was a statistically significant difference in the two groups regarding average TTP strain ( $p$ value $<0.001$ ) and Average TTP SR E ( $p$ value $=0.005$ ), With the MS group having the higher value and the MR group having the lower value and lastly there was no statistical significance comparing average TTP SR S and average TTP SR A in the two groups.(p value $>0.05$ ). 
Table 4. Left atrial size, Left atrial volume index(LAVI), left atrial area change and left atrial ejection fraction (LAEF).

\begin{tabular}{llllll}
\hline \multirow{2}{*}{$\begin{array}{l}\text { Left atrial size, (LAVI), } \\
\text { left atrial area change } \\
\text { and }(\text { LAEF) }\end{array}$} & \multicolumn{2}{c}{ Groups } & \multirow{2}{*}{ T-Test } \\
\cline { 2 - 3 } & MR & & \\
\cline { 2 - 3 } & Mean \pm SD & Mean \pm SD & T & P-value \\
\hline Atrial diameter $(\mathrm{mm})$ & $51.080 \pm 6.670$ & $51.942 \pm 9.792$ & -0.514 & 0.608 \\
LAVI $\left(\mathrm{ml} / \mathrm{m}^{2}\right)$ & $110.652 \pm 38.746$ & $91.572 \pm 27.119$ & 2.853 & $0.005^{*}$ \\
Atrial area change $\left(\mathrm{cm}^{2}\right)$ & $7.342 \pm 3.649$ & $4.176 \pm 1.820$ & 5.489 & $<0.001^{*}$ \\
Atrial EF \% & $46.810 \pm 12.251$ & $31.418 \pm 7.895$ & 7.468 & $<0.001^{*}$ \\
\hline
\end{tabular}

Data are presented as mean \pm SD or number (percent). Atrial $\mathrm{EF}=$ atrial ejection fraction, LAVI=left atrial volume index.

Table 5. P-A interval in the two groups.

\begin{tabular}{lllll}
\hline & \multicolumn{2}{c}{ Groups } & T-Test \\
\cline { 2 - 3 } & MR & & \\
\cline { 2 - 3 } Pean \pm SD & Mean \pm SD & P-value \\
P-A interval (msec) & $183.324 \pm 70.934$ & $224.504 \pm 54.874$ & -3.247 & $0.002^{*}$ \\
\hline
\end{tabular}

Data are presented as mean \pm SD or number (percent)

Table 6. Strain and strain rate parameters.

\begin{tabular}{llllll}
\hline Strain and strain parameters & \multicolumn{2}{c}{ Groups } & \multicolumn{3}{c}{ T-Test } \\
\cline { 2 - 3 } & \multicolumn{2}{l}{ MR } & & \\
\cline { 2 - 3 } & Mean \pm SD & Mean \pm & \multicolumn{1}{c}{ t } & P-value \\
\hline Avrg PALS \% & $24.530 \pm 9.400$ & $16.970 \pm 5.238$ & 4.968 & $<0.001^{*}$ \\
Avrg SRS (1/s) & $1.214 \pm 0.539$ & $0.974 \pm 0.381$ & 2.571 & $0.012^{\star}$ \\
Avrg SRE (1/s) & $-1.236 \pm 0.530$ & $-0.730 \pm 0.385$ & -5.463 & $<0.001^{*}$ \\
Avrg SRA (1/s) & $-0.874 \pm 0.413$ & $-0.682 \pm 0.311$ & -2.625 & $0.010^{*}$ \\
Avrg TTP strain (msec) & $362.718 \pm 53.073$ & $413.272 \pm 81.228$ & -3.684 & $<0.001^{*}$ \\
Avrg TTP SRS(msec) & $176.086 \pm 63.374$ & $197.246 \pm 73.487$ & -1.542 & 0.126 \\
Avrg TTP SRE(msec) & $481.962 \pm 67.039$ & $533.888 \pm 107.213$ & -2.904 & $0.005^{*}$ \\
Avrg TTP SRA(msec) & $703.934 \pm 137.759$ & $744.630 \pm 127.247$ & -1.534 & 0.128 \\
\hline
\end{tabular}

Data are presented as mean \pm SD or number (percent). Avrg=average, PALS $=$ peak atrial longitudinal strain, $\mathrm{SR} A=$ strain rate $\mathrm{A}, \mathrm{SR} \mathrm{E}=$ strain rate $\mathrm{E}, \mathrm{SR} \mathrm{S}=$ strain rate $\mathrm{S}, \mathrm{TTP}$ SR A= time to peak strain A, TTP SR E= time to peak strain rate $E$, TTP strain= time to peak strain,TTP SR $S=$ time to peak strain rate $S$.

\section{Correlation between LAVI and the strain and strain rate parameters}

As shown in (Table 7).

1- In the MR group: There was an average inverse correlation between the LAVI and : Average PALS $(r=-0.898, p<0.001)$, Average SR $S(r=-0.927, p<0.001)$, Average SRE $(r=-0.938, p<0.001)$, Average SR A $(r=-0.957, p<0.001)$ and Average TTP strain $(r=-$ $0.943, \mathrm{p}<0.001)$.

For the correlation of the SR E and the SR A the "absolute values" (i.e., the magnitude of a numerical value without regard to its sign) was used as the negative sign preceding strain and strain rate values are only an indication of shortening or contraction.

2-In the MS group: There was no correlation in all parameters with ( $p$ value $>0.05$ ).

\section{Discussion}

Speckle tracking echocardiography (STE) is a non-Dopplerbased method for the objective quantification of myocardial deformation from standard bi-dimensional data sets, and it allows to obtain the quantification of longitudinal myocardial LA deformation dynamics [11].

The aims of this study were to characterize LA reservoir, conduit, and contractile function using 2D speckle-tracking deformation imaging in patients with severe organic MR and MS and to compare the effect of these two diseases on the left atrial functions.

\section{PA interval}

It was significantly prolonged in the MR group and even more prolonged in the MS group though MS and MR groups had 
Table 7. Correlation between LAVI and the strain and strain rate parameters.

\begin{tabular}{|c|c|c|c|c|c|c|}
\hline \multicolumn{7}{|c|}{ Correlations } \\
\hline \multirow{3}{*}{$\begin{array}{l}\text { LAVI and the strain and } \\
\text { strain rate parameters }\end{array}$} & \multicolumn{6}{|c|}{ LAVI (ml/m2) } \\
\hline & \multicolumn{2}{|l|}{ ALL } & \multicolumn{2}{|l|}{ MR } & \multicolumn{2}{|l|}{ MS } \\
\hline & $\mathbf{r}$ & $P$ value & $\mathbf{r}$ & $P$ value & $\mathbf{r}$ & P value \\
\hline Avrg PALS \% & -0.433 & $<0.001^{\star}$ & -0.898 & $<0.001^{\star}$ & -0.022 & 0.878 \\
\hline Avrg SRS (1/s) & -0.560 & $<0.001^{\star}$ & -0.927 & $<0.001^{\star}$ & -0.170 & 0.237 \\
\hline Avrg SRE (1/s) & -0.611 & $<0.001^{\star}$ & -0.938 & $<0.001^{\star}$ & 0.162 & 0.261 \\
\hline $\operatorname{Avrg}$ SRA (1/s) & -0.675 & $<0.001^{*}$ & -0.957 & $<0.001^{\star}$ & -0.067 & 0.644 \\
\hline Avrg TTP strain (msec) & -0.538 & $<0.001^{\star}$ & -0.943 & $<0.001^{\star}$ & -0.140 & 0.332 \\
\hline Avrg TTP SRS(msec) & 0.014 & 0.888 & 0.163 & 0.257 & -0.064 & 0.661 \\
\hline Avrg TTP SRE(msec) & -0.085 & 0.401 & -0.085 & 0.558 & 0.060 & 0.678 \\
\hline Avrg TTP SRA(msec) & 0.051 & 0.613 & 0.060 & 0.677 & 0.160 & 0.266 \\
\hline
\end{tabular}

Data are presented as mean \pm SD or number (percent). Avrg=average, PALS= peak atrial longitudinal strain, $\mathrm{SR} A=$ strain rate $\mathrm{A}, \mathrm{SR} E=$ strain rate $\mathrm{E}, \mathrm{SR} \mathrm{S}=$ strain rate $\mathrm{S}$, TTP SR A= time to peak strain A, TTP SR E= time to peak strain rate $\mathrm{E}$, TTP strain= time to peak strain,TTP SR $S=$ time to peak strain rate $S$.

similar left atrial size, reflecting affected electrical functions of the left atrium which was more pronounced in the MS group. This is in agreement with Weijs et al work that reported prolonged PA interval in conditions with increased LA pressure and showed that a prolonged PA-TDI is the most important predictor of new-onset A [12].

\section{Peak atrial longitudinal strain (PALS)}

There was a tendency for the MS group compared to the MR group to have less values of strain and strain rate, this was only statistically significant in the average PALS and not in the three strain rate parameters. PALS reflect the reservoir function and compliance of the left atrium. This suggests that the compliance of the left atrium is far more affected by mitral stenosis than by mitral regurgitation, possibly due to restriction, as a result of fibrosis. This is in agreement with Her et al., who reported higher Global PALS value in MR patients than in MS patients, also reported that in mitral valve disease in general, and specifically in MS rather than MR patients, the LA global strain was significantly correlated with degree of LA fibrosis that was histo-pathologically reported [13].

Also Kuppahally et al., showed that the extent of atrial fibrosis detected with late gadolinium enhancement by magnetic resonance correlates with reduction in atrial strain and SR measured with speckle tracking [14].

Future challenges will include defining an atrial strain and strain rate values that can predict the development of $A F$ and allow the beginning of anticoagulation while the patient is still in sinus rhythm, before arrhythmia actually develops, and thus prevent embolic episodes.

Time to peak strain and strain rate

The average TTP strain and average TTP SR E were significantly shorter in MR compared to MS patients $(p=0.007$ and $p=0.01$, respectively). Though the average TTP SR S, and average TTP SR A tended to be shorter in the MR group than the MS, this was of no statistical significance.

\section{Correlation between LAVI and the strain and strain rate parameters}

In the MR group there was an average inverse correlation, as with higher LAVI values the average PALS, average SR S, average SR E and average SR A were all significantly high. This is concordant with Debonnaire et al. work that $L A$ reservoir strain (PALS) showed significant linear correlation with LAVI [15].

In the MS group there was an average inverse correlation, as with higher LAVI the average SR S showed higher values. There was a weak correlation between LAVI and the rest of the strain and strain rate parameters in both MS and MR groups. The above correlation results suggest that the bigger the left atrium, the worse the strain and strain rate regarding MR patients.

\section{Conclusion}

The Left atrial PALS, TTP strain, and TTP SRE can differentiate between volume overloaded and pressure overloaded left atrium. Pressure overload seems to have a more pronounced effect on LA mechanics.

\section{Lists of Abbreviations}

STE: Speckle tracking echocardiography

PALS: Peak atrial longitudinal strain

SR S: Strain rate $S$

SR E: Strain rate E

SR A: Strain rate $A$

TTP: Strain time to peak strain

TTP SR S: Time to peak strain rate $S$

TTP SR E: Time to peak strain rate $E$

TTP SR A: Time to peak strain A

Avrg: Average

LAVI: Left atrial volume index

Competing interests

The authors declare that they have no competing interests. 
Hemeda et al. Cardiovascular System 2018,

http://www.hoajonline.com/journals/pdf/2052-4358-6-1.pdf

doi: $10.7243 / 2052-4358-6-1$

Authors' contributions

\begin{tabular}{|l|c|c|c|c|}
\hline Authors' contributions & HAH & MSE & ESA & SAE \\
\hline Research concept and design & -- & -- & $\checkmark$ & $\checkmark$ \\
\hline Collection and/or assembly of data & $\checkmark$ & $\checkmark$ & -- & -- \\
\hline Data analysis and interpretation & -- & $\checkmark$ & $\checkmark$ & -- \\
\hline Writing the article & $\checkmark$ & $\checkmark$ & -- & -- \\
\hline Critical revision of the article & -- & -- & $\checkmark$ & $\checkmark$ \\
\hline Final approval of article & -- & -- & $\checkmark$ & $\checkmark$ \\
\hline Statistical analysis & $\checkmark$ & $\checkmark$ & -- & -- \\
\hline
\end{tabular}

Publication history

Editor: Chunyu Zeng, Third Military Medical University, China. Received: 01-April-2018 Final Revised: 31-May-2018

Accepted: 05-Jun-2018 Published: 21-Jun-2018

\section{References}

1. Lozano R, Naghavi M, Foreman K, Lim S, Shibuya K, Aboyans V, Abraham J, Adair T, Aggarwal R, Ahn SY, Alvarado M, Anderson HR, Anderson LM, Andrews KG, Atkinson C, Baddour LM, Barker-Collo S et al. Global and regional mortality from 235 causes of death for 20 age groups in 1990 and 2010: a systematic analysis for the Global Burden of Disease Study 2010. Lancet. 2012; 380:2095-128. | Article | PubMed

2. Zuhlke $L$ and Steer AC. Estimates of the global burden of rheumatic heart disease. Glob Heart. 2013; 8:189-95. | Article I PubMed

3. Karan Sud, Shikhar Agarwal, Akhil Parashar, Mohammad Q. Raza and Kunal Patel et al. Degenerative Mitral Stenosis. Circulation. 2016; 133:1594-1604.

4. Cupido BJ, Peters F and Ntusi NA. An approach to the diagnosis and management of valvular heart disease. S Afr Med J. 2016; 106:39-42. I PubMed

5. Cianciulli TF, Saccheri MC, Lax JA, Bermann AM and Ferreiro DE. Twodimensional speckle tracking echocardiography for the assessment of atrial function. World J Cardiol. 2010; 2:163-70. I Article I PubMed Abstract | PubMed FullText

6. D'Andrea A, Caso P, Romano S, Scarafile R, Cuomo S, Salerno G, Riegler L, Limongelli G, Di Salvo G, Romano M, Liccardo B, lengo R, Ascione L, Del Viscovo L, Calabro $P$ and Calabro R. Association between left atrial myocardial function and exercise capacity in patients with either idiopathic or ischemic dilated cardiomyopathy: a two-dimensional speckle strain study. Int J Cardiol. 2009; 132:354-63. | Article | PubMed

7. Lang RM, Bierig M, Devereux RB, Flachskampf FA, Foster E, Pellikka PA, Picard MH, Roman MJ, Seward J, Shanewise JS, Solomon SD, Spencer KT, Sutton MS and Stewart WJ. Recommendations for chamber quantification: a report from the American Society of Echocardiography's Guidelines and Standards Committee and the Chamber Quantification Writing Group, developed in conjunction with the European Association of Echocardiography, a branch of the European Society of Cardiology. J Am Soc Echocardiogr. 2005; 18:144063. I Article I PubMed

8. Pritchett AM, Jacobsen SJ, Mahoney DW, Rodeheffer RJ, Bailey KR and Redfield MM. Left atrial volume as an index of left atrial size: a population-based study. J Am Coll Cardiol. 2003; 41:1036-43. | Article | PubMed

9. Mor-Avi V, Lang RM, Badano LP, Belohlavek M, Cardim NM, Derumeaux G, Galderisi M, Marwick T, Nagueh SF, Sengupta PP, Sicari R, Smiseth OA, Smulevitz B, Takeuchi M, Thomas JD, Vannan M, Voigt JU and Zamorano JL. Current and evolving echocardiographic techniques for the quantitative evaluation of cardiac mechanics: ASE/EAE consensus statement on methodology and indications endorsed by the Japanese Society of Echocardiography. Eur J Echocardiogr. 2011; 12:167-205. | Article | PubMed

10. Quintana M, Lindell P, Saha SK, del Furia F, Lind B, Govind S and Brodin LA. Assessment of atrial regional and global electromechanical function by tissue velocity echocardiography: a feasibility study on healthy individuals. Cardiovasc Ultrasound. 2005; 3:4. | Article | PubMed Abstract | PubMed FullText

11. Cameli M, Incampo $E$ and Mondillo S. Left atrial deformation: Useful index for early detection of cardiac damage in chronic mitral regurgitation. Int J Cardiol Heart Vasc. 2017; 17:17-22. | Article | PubMed Abstract | PubMed FullText

12. De Vos CB, Weijs B, Crijns HJ, Cheriex EC, Palmans A, Habets J, Prins MH, Pisters R, Nieuwlaat R and Tieleman RG. Atrial tissue Doppler imaging for prediction of new-onset atrial fibrillation. Heart. 2009; 95:835-40. | Article I PubMed

13. Her AY, Choi EY, Shim CY, Song BW, Lee S, Ha JW, Rim SJ, Hwang KC, Chang $B C$ and Chung N. Prediction of left atrial fibrosis with speckle tracking echocardiography in mitral valve disease: a comparative study with histopathology. Korean Circ J. 2012; 42:311-8. I Article I PubMed Abstract | PubMed FullText

14. Kuppahally SS, Akoum N, Burgon NS, Badger TJ, Kholmovski EG, Vijayakumar S, Rao SN, Blauer J, Fish EN, Dibella EV, Macleod RS, McGann C, Litwin SE and Marrouche NF. Left atrial strain and strain rate in patients with paroxysmal and persistent atrial fibrillation: relationship to left atrial structural remodeling detected by delayed-enhancement MRI. Circ Cardiovasc Imaging. 2010; 3:231-9. | Article | PubMed

15. Debonnaire P, Leong DP, Witkowski TG, Al Amri I, Joyce E, Katsanos S, Schalij MJ, Bax JJ, Delgado V and Marsan NA. Left atrial function by two-dimensional speckle-tracking echocardiography in patients with severe organic mitral regurgitation: association with guidelines-based surgical indication and postoperative (long-term) survival. J Am Soc Echocardiogr. 2013; 26:1053-62. | Article | PubMed

\section{Citation:}

Hemeda HA, Abd Elmoneum MS, Al Kishk ES and El Darky SAE. Echocardiographic assessment of the left atrial function in patients with mitral valve disease by two-dimensional speckle tracking. Cardio Vasc Syst. 2018; 6:1. http://dx.doi.org/10.7243/2052-4358-6-1 